\title{
Artificial vs. human intelligence in analytics
}

\section{Do computers outperform analytical chemists?}

\author{
Günter Gauglitz ${ }^{1}$ (D)
}

Received: 6 June 2019 / Accepted: 7 June 2019 / Published online: 25 June 2019

(C) Springer-Verlag GmbH Germany, part of Springer Nature 2019

In 1950, Alan Turing, an English computer scientist, who is widely considered the "father" of theoretical computer science and artificial intelligence (AI), proposed a test to determine whether computers were able to "think". In this test, later called the "Turing test", a third person (the evaluator) follows a conversation between a person and a machine. If this evaluator is not able to distinguish the machine from a human being, then the machine is said to be "intelligent" and to have passed the test.

In 1956, John McCarthy announced a workshop at Dartmouth College on the topic "Artificial Intelligence" in the new fields of computer science, natural language processing, and neural networks. These new fields of research were certainly promoted in the following years by the launch of Sputnik and, as a consequence, the establishment of the Advanced Research Project Agency (ARPA) in the USA, which extended funding beyond physicists and engineers to mathematicians. After ups and downs, AI was considered to be the basis of expert systems in the 1980s. At this stage, AI combined searches of a database with causal relationships, extracted rules, obtained a decision tree, and started self-learning.

AI uses neural networks. Their development began in 1943, and nowadays they allow language processing, information retrieval, speech recognition, robotics, pattern recognition, and data mining. In analytics, the advantages of the application of neural networks were first experienced in sensor experiments. Chemical sensors demonstrated poor selectivity. For this reason, sensor element arrays with modified functionalized polymers were used. Model-based chemometric methods, such as principal component regression or partial least squares, were applied. Frequently, these methods were improved by using model-free neural networks for classification, such as

Günter Gauglitz

guenter.gauglitz@uni-tuebingen.de

1 Institute of Physical and Theoretical Chemistry, Eberhard Karls Universität, Auf der Morgenstelle 18, 72076 Tübingen, Germany
Kohonen approaches, or even quantification based on large training sets (artificial neural networks with back-propagation). It soon became obvious that the quality of an evaluation using neural networks for sensor arrays depended on the size of the training set. The computing power available then and the sample structure of the hidden layer of the "neurons" limited the quality of the results. In parallel, algorithms for searches of spectral libraries and methods for pattern recognition were developed, both becoming of interest in analytics.

Within the last 10 years, computer power has increased exponentially. Nowadays, the former performance of desktop computers is realized in the microprocessors for smartphones. Accordingly, training sets can comprise hundreds of thousands of single graphs, sensor data, or figures. We find that, with machine-learning algorithms, computers learn to understand causal relationships and establish concepts. This means that, through learning by experience, layered structures are generated and unsupervised learning is possible. Data and derived concepts are superimposed in layers; the conceptual learning process forms a multilayer system, and thus, can be implemented through a neural network with a drastically increasing number of layers. Computer science was happy when AI beat the human chess world champion and even succeeded in the more complex game GO. The architectures of such deep neural networks lead to the term "deep learning". Information from the input layer is transmitted to the next layer, which handles this information and transfers it to the next hidden layer. Deep learning is a subset of machine learning.

In former times, it was assumed that a computer could not interpret data beyond the training data. New programming tools; algorithms for self-learning; almost limitless computing power; and the capability of making inferences, generalizations, and analogies open up new domains for neural networks and AI. The computer starts to "think" strategically. Besides applications such as autonomous driving, robotics, and facial recognition (to name just a few), applications in health care are performing diagnoses during surgeries within seconds. They determine the border of a cancerous tumor on an X-ray or even 
in a Raman image. Another possible application is the identification of unforeseen physicochemical interactions between gases in a mixture or of processes in/on the material during catalysis that had not been considered in the training set.

At present, AI requires a huge amount of data to learn something with a success rate comparable to that of humans (child or expert) with far less experience than the AI machine. Self-learning and advanced training allow AI to develop strategies and concepts, to find new inferences, to extract hidden features, and to start making decisions. However, AI has problems in determining the spatial relationship between areas of a picture (pattern recognition) and identifying intentions beyond the training set (such as unforeseen decisions of a pedestrian in autonomous driving). In addition, humans feel empathy, they are creative, they are able to find unconventional approaches, and consider ethics. Computers are unbeatable when it comes to handling big data. However, they first have to learn, whereas humans can perceive. The best ones we call geniuses, whom computers cannot replicate.

Looking at the Turing test, modern AI certainly comes closer to a break-even point. However (to finally turn to analytics), the AI of computers can support analytical chemists, but the experience of analytical chemists, with their capability to identify unconventional approaches, to detect minimal deviations from the normal, and to look far beyond a given "data set" makes them, and therefore, human intelligence, superior to $\mathrm{AI}$, and, in my opinion, will continue to do so in the future. Computers will support analytics to a very high degree through data mining, deep thinking, and advanced control of experiments. Without doubt, AI is highly beneficial for the analytical chemist; however, it will take some time before AI will be able to demonstrate intuitive perception. In my view this means that $\mathrm{AI}$ will support analytical chemists, but cannot replace their human intelligence.

Publisher's note Springer Nature remains neutral with regard to jurisdictional claims in published maps and institutional affiliations.

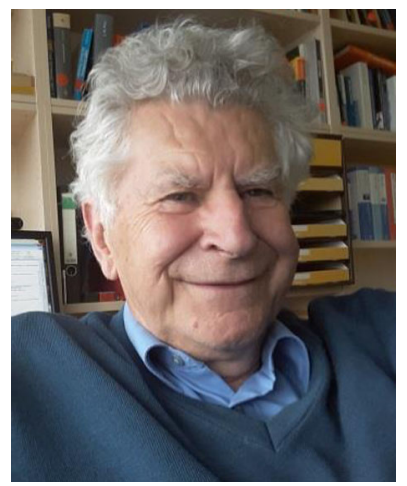

Günter Gauglitz is Senior Professor at the Eberhard Karls University of Tübingen working on analytical and physical chemistry. He was Chairman of the Division of Analytical Chemistry of the German Chemical Society, and chaired the Europt(r)ode VIII meeting. For more than 15 years, his main scientific interests have centered on research and development in chemical and biochemical sensors, with special focus on the characterization of interfaces of polymers and biomembrane surfaces, spectroscopic techniques, use of spectral interferometry to monitor changes in the optical thickness of thin layers, and the effects of Fresnel reflectivity at interfaces. He has been an Editor of Analytical and Bioanalytical Chemistry since 2002. 